\title{
Molecular switches in nanocontainers
}

Yael Diskin-Posnera , Linda J.W. Shimon ${ }^{a}$, Anton Hanopolskyi ${ }^{b}$ and Rafal Klajn ${ }^{b}$

aUnit of Chemical Research Support, Kimmelman Building, The Weizmann Institute of Science, Rehovot 76100, Israel. E-mail: yael.diskin-posner@weizmann.ac.il

${ }^{b}$ Department of organic Chemistry, Kimmelman Building, The Weizmann Institute of Science, Rehovot 76100 , Israel.

Molecular switches, entities that can be toggled between two or more forms upon exposure to an external stimulus, such as light, often require conformational freedom to isomerize. Confining these molecules to volumes only slightly larger than the molecules themselves can profoundly alter their properties. Molecular switching events often entail a dramatic conformational change that require the confining cage to be flexible enough to adapt to the shape of the guest and allow it enough freedom to successfully switch between the different conformers. In the absence of the cage the photoswitching can be either suppressed or the guest can be excluded from the solubilizing medium.

A very flexible, and adapting to accommodate cage synthesized from triimidazole ligand and $\mathrm{Pd}(\mathrm{TMEDA})\left(\mathrm{NO}_{3}\right)_{2}$ receptor [1]. This cage encapsulating molecules such as spiropyran [2] and azobenzene derivatives [3] was reported recently. The crystal structure unit cell volume is relatively large and contains a cage, one or two guest and numerous counter ions and solvent molecules. Current work focused on encapsulating photoswitching molecules such as methyl orange and pyrazol. The crystals of the cage containing these guests are very sensitive to the cryo protectant and pass a single crystal to single crystal transformation. Even after cage conformational changes and the shifting of guest molecules inside, the crystals still diffract and allow a full single crystal $X$-ray structure analysis.

These newly synthesized materials are a novel type of information storage medium in which messages could be written and erased in reversible fashion using light. Furthermore, utilizing photoswitchable molecules for medicinal purposes became one of the modern ways to tune local drug activity or to selectively trigger drug release on site. Water soluble coordinational cages can serve as robust drug-delivery system.

References

[1] Samanta D., Mukherjee S. ,Patil Y.P. \& Mukherjee P.S.; Chem. -A Eur. J. 18, 12322-12329 (2012)

[2] Samanta D., Galaktionova D., Shimon L. J. W., Diskin-Posner Y., Chu Z., Avram L., Král P. \& Klajn R.; Nature Comm. 9, $641(2018)$

[3] Samanta J., Gemen Z., Chu Y. Diskin-Posner Y., Shimon L.J.W. \& Klajn R.; Proc. Natl. Acad. Sci USA 201712787 (2018). Doi: 10.1073/pnas.1712787115 